\title{
Why anything rather than nothing? The answer of quantum mechanics
}

\author{
Vasil Penchev, vasildinev@gmail.com \\ Bulgarian Academy of Sciences: Institute of philosophy and Sociology: \\ Dept. of Logical Systems and models
}

\begin{abstract}
Many researchers determine the question "Why anything rather than nothing?" as the most ancient and fundamental philosophical problem. Furthermore, it is very close to the idea of Creation shared by religion, science, and philosophy, e.g. as the "Big Bang", the doctrine of "first cause" or "causa sui", the Creation in six days in the Bible, etc.

Thus, the solution of quantum mechanics, being scientific in fact, can be interpreted also philosophically, and even religiously. However, only the philosophical interpretation is the topic of the text.

The essence of the answer of quantum mechanics is:

1. The creation is necessary in a rigorous mathematical sense. Thus, it does not need any choice, free will, subject, God, etc. to appear. The world exists in virtue of mathematical necessity, e.g. as any mathematical truth such as $2+2=4$.

2. The being is less than nothing rather than more than nothing. So, the creation is not an increase of nothing, but the decrease of nothing: it is a deficiency in relation of nothing. Time and its "arrow" are the way of that diminishing or incompleteness to nothing.
\end{abstract}

Key words: Creation, dark energy, dark matter, entanglement, quantum information, the "Big Bang”, the Standard model 


\section{"Why anything rather than nothing?"}

Many researchers determine the question "Why anything rather than nothing?" as the most ancient and fundamental philosophical problem (Wippel 2011). Indeed, that problem should be questioned first, before any other one (Hoffman, Rosenkrantz 2010). For example, it should underlie "What or which is the primary substance of the world?", "Spirit or matter?" etc.

The pathway from the being to time was traced by Heidegger in his famous "Sein und Zeit" (1927), where he insisted on the "question of the meaning of the Being" as the beginning of philosophy. We will try to answer it, too, by means of science rather than only by means of philosophy.

Nevertheless, we will find, as Heidegger, the time linked to the being inherently once the question "why the being rather than nothing" is asked in the beginning.

Three from the most fundamental domains of human culture: religion, physics, and mathematics, have offered three quite different versions about the Creation. Furthermore, our problem is very close to the idea of Creation shared by religion, science, and philosophy, e.g. as the "Big Bang", the doctrine of first cause or causa sui, the Creation in six days in the Bible, etc.

In mathematics, the "empty set", a mathematical equivalent of "nothing", is what is in the beginning. It generates the natural numbers, choice, sets and all mathematical objects, complexed or not, on their base. So, mathematics gives an example how its world can be constructed on the ground of "nothing" rigorously.

The concept of creation generates big troubles in physics, which are due to the following. The most fundamental postulate of physics, energy conservation, can be called a "no creation" axiom:

Energy must conserve always, and thus anything physical, having nonzero energy in definition, cannot appear from nothing possessing zero energy: for this would violate energy conservation.

Furthermore, time is thought in two opposite ways, inconsistent to each other, in different physical theories: either reversible (classical mechanics, relativity, etc.), or irreversible (thermodynamics, etc.).

The solution of statistical thermodynamics about time is very interesting and successful. It managed to resolve the contradiction of reversible time in mechanics and irreversible time in thermodynamics. The thermodynamic irreversibility according to it is a result of the statistical averaging of mechanical reversibility. A huge part of information is lost after averaging, and just that loss generates irreversibility in thermodynamics. The loss of information in thermodynamics can be generalized by the notion "hidden variables", hidden by, and after averaging.

Quantum mechanics was that domain of physics which generates new fundamental questions about the relevant way how to be reconciled both reversible and irreversible time unto a single consistent scientific theory. Quantum mechanics was forced to introduce the Planck constant, which is thermodynamic in essence, as fundamental to mechanical motion. Thus, it should reconcile the reversible time of mechanics with the irreversible time of thermodynamics already in its foundation.

Many scientists, even Einstein, expected that its solution should be similar to that of statistical thermodynamics. However, that conjecture turned out to be fundamentally wrong:

"No hidden variables in quantum mechanics!" might be the "slogan" of the solution about the reversible and irreversible time in quantum mechanics. Anyway, the solution of quantum mechanics is partly analogical to that of statistical thermodynamics. A huge part (exactly the half) of information is 
lost in any single measurement ${ }^{1}$. However, that loss is not due to averaging or to human ignorance. It is a fundamental law of nature due to the limitation imposed by the Planck constant. That fundamental loss is caused by the course of time in the final analysis. All rejected counterfactual alternatives might represent that necessary loss of information.

There exist two different, and maybe even inconsistent conjectures in physics about the "creation":

The "Big Bang" is the most popular one. It postulates a special point of the creation (the "singularity" in " $\mathrm{t}=0$ "), in which physical laws do not hold yet. However, they (first of all, both energy conservation and reversible time of relativity) hold at any time thereafter.

The viewpoint of quantum mechanics is different and rather similar to that of Descartes: mainly in the "Third Meditation" (Descartes 1641; Husserl 1931; Мамардашвили 1981; Secada 1990; Gorham 2007, 2008):

The creation is permanent, at any time, and it is due to the irreversibility of time. If one projects all irreversibility of time into a single point of the beginning, the well-known picture of the "Big Bang" will appear.

One can search for empirical confirmations versus the "Big Bang". If the "Big Bang" was real, any physical objects in the universe older than the universe itself would be impossible to exist. However, if the "Big Bang" is not real, but only a hypothetical projection of the irreversibility of time into a single zero point, it may be an averaging of the course of time in all points in the universe. Arbitrary deviations to that average quantity would exist in various spots of the universe. The most objects might be younger than the universe, but at least a few ones should be older than it. The existence of objects older than the universe is partly ${ }^{2}$ confirmed experimentally (Chamberlain, Aller 1951; Spite, Spite 1982; Molaro 1987; Bond et al. 2013).

Various interpretations of the solution of quantum mechanics might exist analogically. Thus, the solution of quantum mechanics, being scientific in fact, can be interpreted also philosophically, and even religiously.

Indeed, the opposite conjecture of the "Big Bang" was elaborated by the Belgian Catholic priest Georges Lemaître $(1927 ; 1931 ; 1946)$, so early as in 1927. It was able to reconcile science (from the "Big Bang" on) with religion (the "Big Bang" itself as God's creation). Analogically, the solution of quantum mechanics admits religious interpretations.

1 In fact, any quantum leap is determined unambiguously by both initial and final state. So, the number of necessary variables is exactly the same as the classical case of smooth motion, and not the half of it: only as kinds of variables are the half of them, but each of them twice: once for the leap initial state, and once more for the leap final state. However and unlike the classical case, that exhausting number of variables is not accessible in any single measurement, but in two ones eventually. Thus, a new problem appears as far as Heraclitus' "No man ever steps in the same river twice, for it's not the same river and he's not the same man" holds. Continuing the metaphor of Heraclitus' "river", quantum mechanics is forced to describe all states of both "river" (i.e. the investigated quantum entity) and "man" (i.e. the apparatus eventually together with the experimenter), which might happen in future: properly, this is the wave function of the the entity at issue. The wave function refers only the half variables in comparison to the classical case, but the information about them is doubled for wave function is complex rather than real. From that viewpoint, hidden variables in quantum mechanics cannot exist for the information is exactly the same as in the classical case. The half of information is lost only after measurement and then secondarily restorable as a probability distribution of all states of both "man" and "river" in a series of measurements.

2 The contemporary accuracy of measurements do not allow for any unambiguous statement: they may or may not be older the universe really. However, the dominating paradigm of the "real Big Bang" influences very strongly onto the formulations: so, all publications emphasize that those objects have appeared very soon after the Big Bang rather than a little before it though the experimental accuracy allows of both kinds of interpretation. 
However, only the philosophical and mathematical interpretation is discussed here. Its essence is: the creation is permanent and due to the irreversibility of time. The creation is mathematically necessary. Thus, it is not due to one's free will (e.g. God's, the observer's, etc.). It is not an addition, but taking away due to the rejection of the reverse "half" of time. The being is less than nothing.

The viewpoint of quantum mechanics about the creation reveals the following. The essence of the answer of quantum mechanics (in physical terms rather than in philosophical notions) is:

The CPT-theorem is fundamental (Bell 1955; Pauli 1955; Luders 1954): it manages the transformation of the discrete charge (electric and color charge, weak isospin) into a space-time position. Thus, it manages how the discrete transformation of elementary particles is equivalent to a continuous space-time trajectory. Weak (or the unified electro-weak) interaction manages the mechanism how the discrete charges can be transformed into space-time trajectories. Weak interaction implies the Higgs mechanism (Englert, Brout 1964; Higgs 1964; Higgs 1964a; Guralnik, Hagen, and Kibble 1964; Glashow 1961; Anderson 1963; Gilbert 1964; Streater 1965; Higgs 1966) generating mass (energy) at rest by cutting the opposite direction of time ${ }^{3}$.

So, mass (energy) at rest represents the total probability of all cut alternatives after the unavoidable choice in the course of time. The antiparticles (with opposite charges) represent the "difference" of the particles to the physical "nothing" (which is not a vacuum, but the result after their annihilation according to the physical meaning of "annihilation"). Thus, the particles identical to their antiparticle (such as photons, $\mathrm{Z}^{0}$ bosons, $\pi^{0}$ mesons) represents the physical nothing from the viewpoint of the physical being (which may be defined as possessing any nonzero mass at rest) ${ }^{4}$.

What the "Higgs mechanism" means moved into the pole of the "First Philosophy" is very simple, but hardly obvious: the nothing is unstable. It breaks down "spontaneously", i.e. by itself. All the being is due to that fundamental instability of the nothing and thus the concept of creation should be underlain by that instability. So, the answer of the question "Why anything ..." is: because the nothing is unstable.

However, the still more fundamental question: “O.K., but why is the „,nothing“ unstable?” seems not to allow a direct physical answer. It needs a relevant mathematical reason revealable in the foundation of mathematics, in set theory, and will be discussed as the mathematical necessity of creation after a few paragraphs.

What the "Higgs mechanism" discusses immediately is how the elementary particles corresponding to the weak and strong fields unlike that of the electromagnetic one acquire their nonzero mass at rest as all experiments confirm. Furthermore, the way for them to acquire their mass at rest implies for all possessing nonzero mass at rest the way to acquire it. Consequently, the Higgs mechanism elucidates how the matter in a physical sense appears always:

\footnotetext{
${ }^{3}$ A complex relation between "choice" (the axiom of choice), the imaginary and real domains of Minkowski space utilized by special relativity, on the one hand, and the complex separable Hilbert space utilized by quantum mechanics, on the other hand, is available. Namely: by means the axiom of choice, the imaginary domain may be mapped isomorphically into that Hilbert space therefore cutting the real domain equivalent to the opposite direction of time or in other words, choosing the imaginary domain of Minkowski space.

4 The "boundary' of the physical nothing between particles and antiparticles is conventional in a sense. Theoretically, any state may be granted as it. In fact, that state is determined unambiguously by the three most fundamental physical constants: the Planck constant, the light speed in vacuum, and the gravitational constant. It may be considered as "zero" only ontologically for those constants imply nonzero physical parameters for it.
} 
That way in the pole of ontology can be represented as follows. Time and its "arrow" is what breaks down the symmetry by rejecting the opposite direction of time ${ }^{5}$. Anyway, all rejected as belonging to the opposite direction of time is represented in the actual course of time as that mass at rest, and this is the way for it to appear from the nothing, i.e. as if a "byproduct" of time.

Quantum mechanics can be considered as a cognitive "microscope" for investigating right the genesis for that asymmetry of time to happen. Indeed, it is a theory for how the irreversible time appear physically from the coherent and reversible quantum state. Both electromagnetic and strong interactions as well as gravity in a sense share CT-symmetry implying P-symmetry as a separate and independent symmetry for the total CPT-symmetry. In that particular framework, only the antiparticles are what represent the opposite direction of time in the coherent "primordial soup" of being, just in the process of how the "arrow of time" appears.

The weak interaction complicates the above picture adding P-asymmetry to C-asymmetry to represent the appearing T-asymmetry in the yet coherent "primordial soup" of the being.

Where is the room of gravity in breaking down the symmetry?

Indeed, there are two different conceptions about mass of rest: the one according to the Higgs mechanism in the process of how it appears; the other, according to general relativity as it interacts. Both "kinds" of mass at rest should be equal to each other in a generalization of the "equality of inertial and gravitational mass": the former in the Higgs mechanism, and the latter in general relativity.

An approach to both Higgs and gravitational mass at rest (and their eventual unification) is the following: to be discussed the way for the opposite (rejected) direction of time to be represented in general relativity:

One has to start from special relativity, in which the "normal" direction of time is represented by the subliminal (or "imaginary') domain in Minkowski space, and the "opposite" direction, by the superluminal (or "real") one. The change of the direction of time means the exchange the two domains as well as the T-symmetry.

If one utilizes the conjecture that general relativity can be considered as the generalization of special relativity right as to the superluminal domain (Penchev 2013), the change to the opposite direction of time means involving the subluminal domain of pseudo-Riemannian space for the superluminal one of Minkowski space. In the pole of ontological reflection, this means that all information lost for rejecting the opposite direction of time is represented anyway in the "normal" course of time, however in the total form of mass (energy) generated by the transformation of Minkowski space into pseudo-Riemannian space and resulting into gravitational interaction.

As the transition from the normal for us to the opposite direction of time, as the reverse transition from the opposite to the normal for us direction of time, results into one and the same pseudo-Riemannian space, into one and the same general relativity. Mass and energy in general relativity are only positive and generate only attraction: the change of the direction of time does not imply antigravity: it seems not

\footnotetext{
5 The physical meaning of that ontological viewpoint is the following. The violation of symmetry is forced by the different physical dimensionality of Minkowski space corresponding to the light speed in vacuum, and thus to "speed", and of the utilized Hilbert space corresponding to the Planck constant, and thus to "action". The gravitational constant is what adds a second equation to the relation of their dimensionality, therefore determining that violation of symmetry unambiguously. As far as the real domain of Minkowski space, equivalent to the opposite direction of time, is cut for the transform of the former into the latter space, one may say that the cut direction of time is represented implicitly in the gravitational constant and then, by any mass involving in gravitational interaction.
} 
to exist. Following CT-symmetry, one may notice that the mass of all antiparticles is identical to that of the corresponding particles, their counterparts.

Thus, general relativity discusses the ultimate result after the direction of time is established as the normal one for us. On the contrary, quantum mechanics means the "primordial soup" of a partly in general coherent state, in which both directions of time co-exist, and the asymmetry of time, "arrow of time" is yet in the process of its constitution and obeys CPT-invariance. Thus, the viewpoint of general relativity is disjunctively alternative to that of quantum mechanics as well as equivalent to it in a sense.

However, the choice of the opposite direction of time leads to the same universe identical to ours. All antiparticles exist only in the "primordial soup" of quantum coherent states. All of them result into the total mass (energy) in the normal course of the ultimate "arrow" of time. The C-symmetry, T-symmetry, and P-symmetry are decomposed from the "primordial" total CPT-symmetry and even, each of those three symmetries is reduced to idempotency: the "antiparticles" coincide with the particles; the opposite direction of time coincide with the normal one, and space is isotropic.

Electromagnetism can visualize that transition if one admits both electric charges situated just "before the boundary" of the ultimate "arrow" of time, and the single magnetic charge as the result of the identification of both electric charges just "after the same boundary". That illustration can serve as a metaphor of how the Higgs mechanism in the "primordial soup" of time is transformed into the mass (energy) of general relativity after the ultimate constitution of the "arrow" of time.

\section{The "dark" problems}

However, there exist two huge "dark" problems concerning the equality or equivalence of the Higgs mechanism to the mass (energy) of general relativity: "dark matter" and "dark energy":

"Dark matter" (Trimble 1987: 451-452) consists in the experimentally very well corroborated fact (Ade 2016) that the angular speed of rotation of huge celestial objects such as our galaxy, the Milky Way, exceeds many times the speed allowed according to the total mass of the visible matter in the object at issue (e.g. the Milky Way). In other words, almost all huge celestial objects such as galaxies, star clusters, nebulae, etc. should break down and flush in all directions in virtue of the centrifugal forces of their rotations. However, nothing like this is observed. The only possible, or at least the most probable explanation according to the contemporary science, is the availability of hidden or "dark" mass and matter, which is not visible until now or even in principle. Furthermore, this hidden matter should possesses about 5.47 times more mass than the usual, visible mass. Of course, this conclusion is shocking since it means that our physical cognition refers only to a relatively insignificant part (about 18.3\%) of the universe. Anyway, all experimental observations confirm that fact.

All mass due to the Higgs mechanism as well as all elementary particles are observable. Unlike all those, the dark matter is revealable only by its gravitational effect preventing the action of centrifugal forces.

"Dark energy" (Riess et al. 1998: 1009) consists in very well confirmed fact ${ }^{6}$ that the expansion of the universe is accelerated: the speed of its expansion increases permanently. This means according to the level of contemporary physical cognition that some unknown huge amount of energy pours into universe at any moment of time. That is properly the "dark energy".

6 The Dark Energy Survey: https://www.darkenergysurvey.org/the-des-project/overview/ 
Both dark energy and dark mass are "dark": this means that their existence is established only indirectly by means of their effect, but they seem not to be directly observable at least until now. However, the dark matter is a static "dark" effect, while the dark energy is a dynamic one.

The total amount of dark energy in the universe is a few times more than dark matter and more than ten times more than the visible matter and energy. The experimentally confirmed proportions are: visible matter and energy is $4.9 \%$; dark matter, $26.8 \%$; dark energy, $68.3 \%$. So, the existence of dark energy is even more striking than that of dark matter: one may use the metaphor that the "shore of our knowledge" is much less than the "ocean of our ignorance" about what are both dark matter and dark energy.

The attempts to be explained those "dark" phenomena might be distributed into two basic groups: "standardly", by means of the Standard model; or "non-standardly", by means of theories or experimentally observed phenomena out of the framework of the Standard model.

Confinement or "color confinement" is one of the main conjectures among the standard explanations of the dark phenomena. It means a mysterious, hypothetical force which holds on the quarks linked very strongly to each other and preventing for them to be observed stand alone. The hypothesis is that it increases (even exponentially) proportionally at a distance unlike all known and observable forces in nature decreasing at a distant.

Here is how the confinement might explain dark energy:

The expansion of the universe is a process of positive feedback since the expansion itself generates energy by means of the mechanism of confinement. In other words, the dark energy is equivalent to the expansion of the universe and thus, it is self-accelerating by itself. Then, the dark matter might be a static phenomenon of dark energy, due to the current amount of energy in any huge rotating celestial object such as our Milky Way.

The main objection is that the degree of tension due to color confinement has an upper limit, after which the color interaction breaks, just as an over-extended elastic spring breaks, therefore a "colorless jet" of hadrons appears: the so-called hadronization of quarks or gluons. Anyway, that "jet" tends to expand further and further in time because the color interaction in any new colorless hadron has been extending in turn until it "breaks", too, and generates more new quarks or gluons, which are hadronized again immediately and unobservably ${ }^{7}$.

Our key question should be what happens with energy conservation during the process of hadronization: the energy of the "broken over-extension" passes into the mass-energy of the generated ("from the vacuum") anti-counterparts for any quark or a pair of quark and antiquark for any gluon. This can explain how the space extension itself can generates energy by itself by means of color elementary particles such as quarks or gluons and the extraordinary property of color confinement transforming the extension of the universe into a kind of "perpetuum mobile".

The process of hadronization may throw light furthermore to the intimate mechanism of how time appears from space over the upper limit of its allowed extension. The space extension generates energy for color confinement. That space extension breaks over a certain energetic bound and a hadron jet appears. Any element of the hadron jet turns out to be doubled by a pair of quark and antiquark, and hadronization obeys the strong, first of all, and electromagnetic interaction. Both are CT-invariant, and

\footnotetext{
${ }^{7}$ The only exception is the top quark, whose time of decay is supposed to be less the time necessary for its hadronization, so the products of its decay can be observed and thus indirectly, the top quark itself as if "bare" (Abachi et al. 1995; Abe et al. 1995).
} 
P-symmetric because of CPT-invariance. As all the Standard model, they are Lorentz-invariant, which is very closely linked to the CPT-invariance. Thus, any pair quark-antiquark can be considered equivalently as a single particle doubled in both directions of time ${ }^{8}$. Those "two directions of time" appear for exceeding the upper limit of allowed space extension: time seems to appear for the limitation of spatial "elastic" extension".

There are at least a few objections against color confinement as a possible source of dark matter and energy:

It is yet only an ad hoc empirical hypothesis explaining very well a series of phenomena studied by quantum chromodynamics, but it cannot be deduced mathematically from the formalism of quantum mechanics and quantum chromodynamics. It is corollary from one from the seven "Millennium Prize Problems", namely the "Yang-Mills and Mass Gap" problem (http://www.claymath.org/millenniumproblems).

"Color confinement" is not described yet quantitatively and theoretically well enough, but rather qualitatively. Thus, the origin of the energy transformed into hadrons after hadronization is not elucidated: rather that kind of energy is only postulated ad hoc for the abundance of corroborating experimental data.

Furthermore, there exist too essential problems about untarity or Lorentz invariance of hadronization. The main "nonstandard" conjecture about dark matter or dark energy is the phenomenon of entanglement.

Entanglement is a phenomenon forecast yet by Einstein (together with Podolsky and Rosen) in 1935, in a very, very famous article (Einstein, Podolsky, and Rosen 1935) as the refutation of the completeness of quantum mechanics, and independently by Schrödinger in the same year (Schrődinger 1935). Now, entanglement is very well corroborated experimentally ${ }^{10}$.

In Einstein's words, it is a "spooky action at a distance" meaning that it should be neither Lorentz invariant nor unitary. Roughly speaking, one may say that it transfers only "pure" information about a certain quantum state (called "quantum information") at any distance instantly without any carrier possessing a certain nonzero amount of mass or energy. Even more mysterious: any other elementary particle such as an electron or a photon changes its state after it has obtained that "secret message" from its entangled counterpart(s). This seems as if an electron or a photon, or any other elementary particle has "free will" making a decision about how to change its state after the quantum message has been obtained by it.

\footnotetext{
8 One may figure motion at a velocity as the elastic extension of space. Then, the speed of light is the upper bound: a limit, after which the space is "ruptured" into parts, e.g. into particles such as an electron and a positron. On the contrary, the Plank constant is the minimal possible action between two or many parts, under which those "parts" (ostensibly) are a single whole necessarily. So, both speed of light in vacuum and Planck constant are boundaries between discreteness and continuity in a physical and thus experimental sense. Nonetheless, they are absolutely independent of each other, even their physical dimensionalities are different. One may imagine further the special case where the one criterion for discreteness to be satisfied, but the other one not. The quarks unable to exist alone seem to fall right into that intermediate or "conflict" area between the two different boundaries separating discreteness and continuity. As far as they interact with each other and can interact with other elementary particles both by strong and by electromagnetic interaction, the Planck constant seem to be exceeded, but the speed of light not in the special case of quarks. This seems to be the essence of confinement from the present viewpoint.

9 The pair of a quark and an antiquark may be interpreted as rupturing the space by exceeding the "second" barrier, namely that of light speed, and thus rupturing the intimate link of the two directions of time, by which time appears properly. As far as all quarks in definition (or in virtue of confinement) cannot exceed that second barrier, they vanishes instantly either by hardronization (most often) or by decay (only the top quark).

10 The number of confirming experiments is huge (a brief overview e.g. in: Wiseman 2015).
} 
There exist even two "free will theorems" in quantum mechanics (Conway, Kochen 2006; 2009) stating that if the experimenter such as a human being possesses that "valuable commodity" of free will, this implies that any electron or any quantum entity, with which the experimenter deals, possesses the same "valuable commodity" necessarily.

The same idea (about the "free will of an electron") made Einstein to declare in a letter to Max Born that he would prefer to be a "croupier" or a "shoemaker" rather than a physicist if this would be true (Born 1969: 118). The cited theorems state right the "free will of an electron" expressively. So, Einstein ... is possible not to possess "free will" for deciding what to be his profession (as a not less paradoxical solution of the problem).

However, a much more ordinary interpretation of entanglement, without any curious, pictorial, or even ridiculous presentations as above, is possible.

Entanglement is a new form of physical interaction which is both non-unitary and Lorentz noninvariant, and generalizes the concept of physical interaction to certain physical instantaneous actions at any distance (i.e. in a zero time or on other words, as if out of time), and (quantum) information is equivalent to physical action as far as the Planck constant exists.

So, the electron does not "decide" how to change its state after having been obtained the corresponding (quantum) information, but this information changes its state both directly and instantaneously being equivalent to physical action by itself. What seems as "free will" is the direct physical action of information for the information is the quantity of choices (and indeed, measured in units of elementary choices, what the bits are), and therefore information can be considered as the "quantity of free will" in a sense.

All three interactions (electromagnetic, weak, and strong) in the Standard model are both unitary (energy conservation) and Lorentz invariant (not exceeding the speed of light in vacuum). On the contrary, entanglement, if one considers it as a new physical interaction, is neither unitary (its energy is indefinite) nor Lorentz invariant (it is instantaneous, therefore exceeding the speed of light in vacuum).

The only other known physical interaction, which is neither unitary nor Lorentz invariant, is gravity according to general relativity, which is the best confirmed theory of gravity even without any "anomaly".

Indeed, it is not unitary for energy does not conserve (what is conserved according to general relativity is energy-momentum, and therefore the energy itself is indefinite in general). Indeed, it is not Lorentz invariant because the pseudo-Riemannian space of general relativity is arbitrarily (and even differently at any point in general) "curved" to the Minkowski space of special relativity.

Maybe Bronstein (Бронштейн 1936) was the first who demonstrated that gravity can be only locally quantizable since it is only locally unitary and Lorentz invariant, which is due to its continuity and smoothness, but globally not in general. According him, only weak gravitational field is approximately quantizable, and any gravitational field out of all singularities is "weak" locally.

At last, one may build a one-to-one mapping, possessing a clear physical meaning, of the superluminal ("real") domain of Minkowski space into the subluminal ("imaginary") domain of pseudo- 
Riemannian space (Penchev 2013). The sense of that mapping is the interpretation of gravity as entanglement and vice versa ${ }^{11}$.

Both dark matter and dark energy are revealed by gravitational phenomena for very huge celestial objects, the behavior of which is described by general relativity. So, if one may equate entanglement to gravity, entanglement turns out to be a possible source of dark matter or energy, e.g. as the corresponding equivalent mass at rest and gravitational energy. Indeed, entanglement satisfies the condition of being "dark" for it is out of the Standard model describing all "visible".

The explanation of dark energy by means of entanglement can be the following:

The process of decoherence of any entangled state would mean the disappearance of entanglement and its degree of non-unitarity (or Lorentz non-invariance) equivalent to a certain mass (energy) in terms of general relativity. That energy can be called "energy of decoherence" therefore specific for any entangled object and "emitted" in space at any time in virtue of the decoherence itself. That energy is "pure" without any source in the framework of the Standard model for entanglement is out of it, and therefore being "dark". Even more, it is "dark" in principle as well, for it has not any carrier in definition, originating directly from quantum information equated to a physical action by means of the Planck constant.

The equivalence of gravity and entanglement by general relativity is the necessary condition for that explanation of dark energy. If it is accepted as true, dark matter would correspond to the current degree of entanglement as an equivalent amount of mass at rest also out of the Standard model unlike the Higgs mechanism ${ }^{12}$, and directly (non-unitarily and Lorentz non-invariantly) originating from quantum information, too. This means that the process of decoherence transforms dark matter into dark energy as matter and energy are equated to each other by either special or general relativity: the amount of dark mass should decrease by the same amount, by which the amount of dark energy increases.

\footnotetext{
11 The usual interpretation of entanglement in terms of general relativity (e.g. Jensen, Karch 2013) is as, or by means of "wormhole" in space-time (Einstein, Rosen 1935). If one means namely that kind of usual interpretation, any quantum leap in space-time corresponds to a certain, "straight" wormhole "through" space-time. From our viewpoint, this wormhole is equivalent to a certain curvature in space-time (representable by a space-time tensor) and thus to a certain mass-energy (representable by an energy-momentum tensor equated to the former by the Einstein field equation) generating that spacetime curvature. A certain common measure of both straight wormhole and curvature (e.g. and very roughly speaking, their length) has to be the same for that equivalence to hold. The concept, for example, of "holographic duality" means something similar in the framework of that met interpretation of entanglement as wormhole.

12 The Higgs mechanism seems to correspond to the gravitational constant and thus to the unambiguous determination of the relation of Minkowski space and Hilbert space at issue. On the other hand, their relation (or difference) consists mathematically in two members: (a) the real domain of Minkowski space; (b) the axiom of choice for the discretization of Minkowski space to the separable complex Hilbert space as an equivalent. To be "equated" those two members, three physical members known until now and possibly partly or thoroughly overlapping each other should appear: gravitational constant \& general relativity as well as entanglement. If one grants dark matter \& energy to entanglement (as us), it is logically necessary either the Higgs mechanism to allow for a "dark part" (out of the Standard model) or it to be complemented by an additional, yet unknown part to the difference between Minkowski and Hilbert space in question. The problem seems to be directly linked to that of the "cosmological constant" (Einstein 1918) or "Mach's principle" (Einstein 1918: 241) in general relativity, and thus to the expansion of the universe, the "Big Bang" and (ostensibly) "Einstein's biggest blunt" (Gamov 1970: 44). If one admits (as us) that (quantum) information is able to cause physical action out of time (space-time) by entanglement, "Mach's principle" is rejected. Then, the cosmological constant is not necessary, but anyway both possible and consistent as well as the expansion of the universe and even eventually the "Big Bang" as a real event.
} 
Anyway, all physical interactions are able to generate entanglement ${ }^{13}$, and the balance between ordinary and dark matter, and dark energy in the course of time and its change are unclear yet. This needs special theory of mutual transformation between them as well as many observations and experiments before it to be able to be created.

What one may say unambiguously is that dark matter decreases by transforming into dark energy, which increases. However, the amount of new dark matter for new entanglement due to the "visible" interactions remains absolutely unknown, and thus, the general balance and change. Maybe, color confinement takes an essential place in that general balance, furthermore ${ }^{14}$. Anyway, entanglement unlike color confinement is not a process of positive feedback directly, but possibly by the meditation of the "visible" interactions, though hardly, too.

What is to be explained is before the physical creation at any time:

\section{The creation due to mathematical necessity}

One can approach the mathematical necessity of creation as follows. The creation is necessary in a rigorous sense after one has represented mathematically the physical creation by "taking away" as follows:

The operation $A \otimes\{A\}$ (i.e. the generation of a set from a class) means "taking away" and it is possible always, including the application to $\varnothing$ (the empty set) or to another set: $\{A\} \otimes\{\{A\}\}$.

However, the choice from the empty set is not allowed. Thus, choice turns out to be secondary to the natural numbers. They are implied directly and thus, necessarily, from the nothing for choice is implied only indirectly by means of them and by the axiom of choice, which does not include the choice of the empty set. That secondarity of choice is just what implies the necessity of creation for the creation is "before" the choice to appear. The choice appears together with time, however creation is out of time for the creation creates the time among all the rest.

The creation underlain by set theory seems to be leap-like, generating all natural numbers as the result of that necessary leap from nothing into being.

On the contrary, physics describes the same equivalently, but alternatively: as a continuous process of the being to appear, which is observed as the expansion of the universe. In other words, the expansion of the universe is right its appearance as the appearance of the being. However, the result of that process (the natural numbers) is given (or "granted") in advance.

\footnotetext{
13 Indeed, any interaction implies a force acting to any entity participating in the interaction, and thus causes a certain acceleration according to its mass (energy). That acceleration is able to be transformed partly as (or in other words, represented by) different degrees of entanglement between entities in the interaction. In a sense, the entanglement of the interaction is transformed equivalently into the acceleration of the entity at issue, and vice versa. The essence of entanglement and acceleration is one and the same from the viewpoint of quantum information, to which the option of their mutual transformation is due.

14 Confinement and entanglement seem to be similar to each other in a sense. Unlike the usual physical interactions between objects separate in both Minkowski and Hilbert space, the objects interacting either by entanglement or confinement are absolutely separate only in the one of them correspondingly. Entanglement is possible both violating Bell's equation (over the light barrier) or not (below it). The criterion for the partial inseparability of quarks in Minkowski space is their fractional electric charge. At last, one may admit that a phenomenon (e.g. Lewkowycz, Maldacena 2014) as the entanglement of quarks (color entanglement) might exist to complement that scheme of partial separability and inseparability in different senses. That color entanglement might be linked to dark energy, too (e.g. Banerjee et al. 2005).
} 
The creation (and quite particularly: the corresponding "principle of least action" in mechanics) suggests teleology as a certain aim, which is predefined in virtue of its necessity and embodied in the necessary appearance of the natural numbers from the empty set as an equivalent of the "nothing" as to mathematics. In other words, the visibility of teleology can be understood as a "by-product" of the secondarity of the choice.

The operation, which is the generation of a set from the "nothing", implies all natural numbers by the construction described in the "axiom of infinity" in set theory. The set of all natural numbers is infinite, though all natural numbers are finite according to the axiom of induction in Peano arithmetic.

That last circumstance needs elucidation:

First, here is how the axiom of induction implies for all natural numbers to be finite. The unit is finite. Adding a unit to any finite number, another finite number is obtained. If both premises are true, the axiom of induction implies for all natural numbers to be finite.

Furthermore, the set of all natural numbers is infinite though all natural numbers are finite. One may say that the wholeness of all natural numbers, which is meant by the concept of set, implies a new different, "emergent" property, namely to be infinite unlike the natural numbers constituting that wholeness, which are finite. However, as above, that wholeness, maybe as any wholeness, is obtained by taking away rather than by adding to the natural numbers: finiteness turns to be more than infinity just as the finiteness turned out to be less than the "nothing" as the empty set a few paragraphs ago.

One may say the following about the secondarity of choice. Any set may be enumerated (the wellordering principle), which means that it may be mapped one-to-one into some subset of the set of all natural numbers. The empty set can be enumerated by the special natural number "zero". The wellordering principle implies the axiom of choice: this is meant as the secondarity of choice. Thus, the being does not need any choice, free will, subject, God, observer, etc. to appear for it appears in virtue of mathematical necessity.

The creation can be considered as a mathematical truth. The world exists in virtue of mathematical necessity, e.g. as any mathematical truth such as " $2+2=4$ ". However, that fact requires the completeness of the being, which is not demonstrated yet, e.g. as follows:

The operation $A \rightarrow\{A\}$ can be interpreted as a "primary choice", and thus a vicious circle appears. The vicious circle being equivalent to a contradiction generates an empty set, to which it is a characteristic property. That empty set can underlie the successive genesis: "A $\bigotimes\{A\}$ " as a choice means an alternative "A $\rightarrow\{B\} "$ to exist, e.g. $\{B\}=\varnothing$ : that cho ice w ou ld be necessary on ly if $\{A\}=\{B\}=\varnothing$, i.e. "nothing $\rightarrow \varnothing "$ is necessary.

The being is less than nothing in a sense. The being is less than nothing rather than more than nothing. The creation does not add, but takes away. This seems to be paradoxical to common sense. It may be visualized particularly by an example:

Any contradiction (such as $A \wedge \neg A$ as a characteristic property defines the empty set. If one removes either $\mathrm{A}$ or $\neg \mathrm{A}$, a non-empty set in general can be defined by means of either $\neg A$ or A correspondingly as two separated characteristic properties.

Furthermore, the mathematical necessity of the being is consistent to the conception of the "Big Bang", too. We discuss the gradual physical creation at any time, due to the irreversibility of time as mathematically necessary. However, the mathematical necessity of the being underlies the "Big Bang", too, here is how: 
As a few paragraphs ago, the empty set generates the well-ordering of the natural numbers by taking away, or physically, namely the "time", but it has not "started" yet. Time implies energy in virtue of Emmy Noether's theorems (1918). The well-ordering generates the axiom of choice and thus, the choice itself. The "Big Bang" might occur also in virtue of mathematical reasons.

Now, all is ready for the "Big Bang". There are time and energy, though they are not "activated" physically yet. This will be done by the choice, which exists, too. The choice means zero entropy and thus infinite temperature at any finite energy and even possibly, at zero energy. The infinite temperature generates symmetry breakings (such as the Higgs mechanism), and particularly breaks the symmetry of the two directions of time. The latter symmetry breaking starts the time at the moment: $t=0$. The nothing explodes (or "Nothing explodes") by itself by taking away, as the History of the Being.

The creation might be a decrease rather than an increase. The common viewpoint to the creation or to the being is the opposite one. Creation should add rather than take away. The being should be more than nothing ostensibly.

On the contrary, the creation is not an increase of nothing, but the decrease of nothing: it is a deficiency in relation of nothing. You may figure a sculptor who takes away from the stone by the chisel.

Time and its "arrow" take away, for the other direction of time is removed. Time and its "arrow" are the way of that diminishing or incompleteness to nothing. One may represent the nothing as the unification of both directions of time. However, only the one, forward in time, is real. The other one, backward in time, is taken away from the nothing. After the one direction of time has been taken away from that nothing, what remains is right the being. If one could add the reverse direction of time to the being, what would obtain is just the nothing.

The concept of transformation into nothing exists in physics as that of "annihilation". "Annihilation" in physics means the fusion of a particle and its antiparticle into ... light. So, the light (electromagnetic radiation) is the way for us to see the nothing from our viewpoint of the being (i.e. less than nothing). This is the reason for the nothing to be watched as something: namely light, but only from our viewpoint, which is that of "less than nothing".

One might try to interpret some ideas about the "Creation", borrowed by the Bible (Genesis 1:3-4), from the viewpoint of physics:

"And God said, "Let there be light," and there was light.

And God saw that the light was good. And God separated the light from the darkness"15. Our reading of the "light" in both Bible and theory of relativity is the following:

We see the nothing as light because we ourselves are less than nothing. Indeed, the light is an absolute upper border or limit for all being just as the theory of relativity states. To be nothing an upper bound is only another way to say that the being is "negative", i.e. less than nothing.

According to the Bible (John 1:1-4):

"In the beginning was the Word, and the Word was with God, and the Word was God. He was in the beginning with God. All things were made through him, and without him was not any "thing" made that was made" ${ }^{16}$.

15 Cited according to the King James Version:

https://www.biblegateway.com/passage/?search=Genesis+1\%3A3-4\&version=KJV

16 Cited according to the King James Version: https://www.biblegateway.com/passage/?search=John+1\&version=KJV 
Our reading of the "Word" in both Bible and theory of quantum information is: the "Word" is information, particularly quantum information. Ontologically, information, being measured by units of bits, is the quantity of "taking away" from the nothing for the being to be created. Indeed, a bit is the elementary choice between two equally probable alternatives, and thus, right "taking away" the one of them: the other is the chosen one.

Time and information are linked to each other intimately. Information is the quantity of choices measured in units of elementary choices, i.e. bits. Time in turn is the result of choices: the successive series of all chosen alternatives. The first, crucial, and mathematically necessary choice is the choice of the time itself, or in other words, that of the direction of time, or the "arrow of time". Information (the quantity of choices), and time (the series of all results of those choices) are closely linked.

\section{Conclusions:}

\section{Conclusions as negations:}

The state of "nothing" is not stable. The physical nothing is not a general vacuum. The being is less than nothing. The creation is taking away from the nothing. Time is the destruction of symmetry. The creation needs not any (external) cause.

\section{Conclusions as statements:}

The state of nothing passes spontaneously (by itself) into the state of being. This represents the "creation". The transition of nothing into being is mathematically necessary. The choice (which can be interpreted philosophically as "free will") appears necessarily because of mathematical reasons. The choice generates asymmetry, which is the beginning of time and thus, of the physical word. Information is the quantity of choices and linked to time intimately. 


\section{References:}

Abachi, S. et al. (D0 Collaboration) (1995) "Search for High Mass Top Quark Production in pp Collisions at $\sqrt{S}_{\mathbf{S}}=$ $1.8 \mathrm{TeV}$," Physical Review Letters 74 (13): 2422-2426.

Abe, F. et al. (CDF Collaboration) (1995) "Observation of Top Quark Production in pp Collisions with the Collider Detector at Fermilab," Physical Review Letters 74 (14): 2626-2631.

Ade, P. A. R. et al. (Planck Collaboration) (2016) "Planck 2015 results. XIII. Cosmological parameters," Astronomy and Astrophysics 594: A13(63).

Anderson, P. W. (1963) "Plasmons, Gauge Invariance, and Mass<" Physical Review 130 (1): 439 - 442.

Banerjee, S. et al. (2005) "Cosmological dark energy from the cosmic QCD phase transition and colour entanglement" Physics Letters B 611 (1-2): 27-33.

Bell, J. S. (1955) "Time reversal in field theory," Proceedings of the Royal Society of London A 231 (1187): 479-495.

Bond, H.E. et al. (2013) "HD 140283: A Star in the Solar Neighborhood that Formed Shortly After the Big Bang," The Astrophysical Journal Letters 765 (1): L12.

Born, M. (1969) Albert Einstein Max Born Brief-wechsel 1916 - 1955 (kommentiert von Max Born). München, Nymphenburger, p. 118 (Brief № 48/29.04.1924).

Chamberlain, J. W., Aller, L. H. (1951) “The Atmospheres of A-Type Subdwarfs and 95 Leonis," Astrophysical Journal 114 (1): 52-72.

Conway, J., Kochen, S. (2006) “The Free Will Theorem," Foundations of Physics 36 (10): 1441-1473.

Conway, J., Kochen, S. (2009) The Strong Free Will Theorem. Notices of the AMS 56 (2) 226-232.

Gamow, G. (1970) My world line: an informal autobiography. New York, Viking Press.

Descartes, R. (1641) Meditations on First Philosophy with Selections from the Objections and Replies (transl. M. Moriarty), “Oxford World's Classics,” Oxford, University Press, 2008.

Einstein, A. (1918) "Prinzipielles zur allgemeinen Relativitätstheorie. Annalen der Physik," 55 (4): 241-244.

Einstein, A. Podolsky, B., and Rosen, N. (1935) "Can Quantum-Mechanical Description of Physical Reality Be Considered Complete?” Physical Review 47 (10): 777-780.

Einstein, A., Rosen, N. (1935) "The Particle Problem in the General Theory of Relativity," Physical Review 48 (1): 73-77.

Englert, F., Brout, R. (1964) "Broken symmetry and the mass of gauge vector mesons," Physical Review Letters 13 (9): 321-323.

Gilbert, W. (1964) "Broken Symmetries and Massless Particles," Physical Review Letters 12 (25): 713-714.

Glashow, S. L. (1961) "Partial symmetries of weak interactions," Nuclear Physics 22 (4): 579-588.

Goldstone, J., Salam, A., and Weinberg, S. (1962) "Broken symmetries," Physical Review 127 (3): 965-970.

Gorham, G. (2007) “Descartes on Time and Duration," Early Science and Medicine 12 (1): 28-54.

Gorham, G. (2008) “Descartes on God's Relation to Time," Religious Studies 44 (4): 413-431.

Guralnik, G. S., Hagen, C. R. and Kibble, T. W. B. (1964) "Global conservation laws and massless particles," Physical Review Letters 13 (20): 585-587.

Heidegger, M. (1927) Sein und Zeit. Gesamtausgabe, Bd. 2, Frankfurt AM, Vittorio Klostermann, 1977.

Higgs, P. W. (1964) "Broken symmetries and the masses of gauge bosons," Physical Review Letters 13 (16): 508-509.

Higgs, P. W. (1964a) Broken symmetries, massless particles and gauge fields," Physics Letters 12 (2): 132-133.

Higgs, P. W. (1966) "Spontaneous symmetry breakdown without massless bosons," Physical Review 145 (4): 1156-1163.

Hoffman, J., Rosenkrantz, G.S. (2010) Substance, its nature and existence, London, Routledge.

Husserl, E. (1931) Cartesianische Meditationen und Pariser Vorträge, Den Haag, Nijhoff, 1950. 
Jensen, K., Karch, A. (2013) "Holographic Dual of an Einstein-Podolsky-Rosen Pair has a Wormhole," Physical Review Letters 111 (21): 211602(6).

Lemaître, G. (1927) "Un Univers homogène de masse constante et de rayon croissant, rendant compte de la vitesse radiale des nébuleuses extra-galactiques," Annales de la Société scientifique de Bruxelles, Sèrie A 47: 4959.

Lemaître, G. (1946) L'Hypothèse de l'Atome Primitif: Essai de cosmogonie. Neuchâtel/ Griffon/ Paris, Dunod.

Lemaître, G. (1931) "The Beginning of the World from the Point of View of Quantum Theory." Nature 127 (3210), 706.

Lewkowycz, A., Maldacena, J. J. (2014) "Exact results for the entanglement entropy and the energy radiated by a quark," Journal of High Energy Physics 2014 (5): 25.

Luders, G. (1954) "On the Equivalence of Invariance under Time Reversal and under Particle-Antiparticle Conjugation for Relativistic Field Theories," Kongelige Danske Videnskabernes Selskab. Matematisk-Fysiske Meddelelser 28 (5): 1-17.

Molaro, P. (1987) "Upper limit to the boron abundance in the population II star HD 1402," Astronomy and Astrophysics 183 (2): 241-246.

Noether, E. (1918) "Invariante Variationsprobleme," Nachrichten von der Gesellschaft der Wissenschaften zu Göttingen, Mathematisch-Physikalische Klasse 1918: 235-257.

Pauli,Riess, A. et al. (Supernova Search Team) (1998) "Observational evidence from supernovae for an accelerating universe and a cosmological constant," Astronomical Journal 116 (3): 1009-38.

Pauli, W. (1955) "Exclusion principle, Lorentz group and reflection of space-time and charge," in: W. Pauli (ed.) Niels Bohr and the development of physics, New York, McGraw-Hill, pp. 30-51.

Penchev, V. (2013) "The Kochen - Specker theorem in quantum mechanics: A philosophical comment," Philosophical Alternatives 2013 (1): 67-77 (Part 1); 2013 (3): 74-83 (Part 2).

Riess, A. et al. (Supernova Search Team) (1998) "Observational evidence from supernovae for an accelerating universe and a cosmological constant," Astronomical Journal 116 (3): 1009-38.

Schrödinger, E. (1935) “Die gegenwärtige situation in der Quantenmechanik," Die Naturwissenschaften 23 (48), 807-812; 23 (49): 823-828; 23 (50): 844-849.

Secada, J.E.K. (1990) “Descartes on Time and Causality," The Philosophical Review 99 (1): 45-72.

Spite, F., Spite, M (1982) "Abundance of lithium in unevolved halo stars and old disk stars - Interpretation and consequences," Astronomy \& Astrophysics 115 (2): 357-366.

Streater, R. F. (1965) "Spontaneously broken symmetry in axiomatic theory," Proceedings of the Royal Society London A 287 (1411): 510-518.

Trimble, V. (1987) "Existence and nature of dark matter in the universe," Annual Review of Astronomy and Astrophysics 25: 425-472.

Wippel, J. F. (2011) The ultimate why question: why is there anything at all rather than nothing whatsoever? Washington, D.C., Catholic University of America Press.

Wiseman, H. (2015) “Quantum physics: Death by experiment for local realism,” Nature 526 (7575): 649-650.

Бронштейн М. П. (1936) “Квантование гравитационных волн," Журнал экспериментальной $и$ теоретической физики 6: 195-236.

Мамардашвили, М. (1981) Картезианские размышления (январь 1981 г., под редакции Ю. П. Сенокосова.), Москва, Прогресс, 1993. 